\title{
Diffractive beam splitting microlens for fiber coupling fabricated by UV imprinting process
}

\author{
Jauh Jung Yang ${ }^{\mathrm{a}}$, Chi-Feng Chen ${ }^{\mathrm{b}}$, Yunn-Shiuan Liao ${ }^{\mathrm{a}, *}$ \\ a Department of Mechanical Engineering, National Taiwan University, Taipei, Taiwan, ROC \\ ${ }^{\mathrm{b}}$ Department of Mechanical Engineering and Institute of Opto-Mechatronics Engineering, National Central University, Chung-li, Taiwan, ROC
}

Received 8 August 2007; accepted 25 September 2007

\begin{abstract}
A polymeric diffractive microlens (DML) for coupling the incident light to two individually plastic optical fibers was designed and fabricated by imprinting process. The DML pattern of this dual-focal-point DML was designed with an optimal rotation-angle and realized by template fabrication and UV imprinting. Tetraethoxysilane oxide etching was developed and employed in the template fabrication process to minimize the related processing errors such as misalignment and dimension accuracy. A modified imprinting process is proposed in the experiment; DML with flexible polyethylene terphthalate substrate was imprinted by roller. After curing and demolding, the DML structure was successfully duplicated on polyethylene terphthalate substrate. The prototype was inspected and to confirm the optical properties and the results showed that the diffraction efficiency is $51 \%$ (designed value is $57 \%$ ) and the uniformity error is only $1.5 \%$.

(C) 2007 Published by Elsevier B.V.
\end{abstract}

Keywords: Beam splitter; Diffraction microlens; UV Imprinting

\section{Introduction}

Fiber-to-the-home (FTTH) systems have been expected to be the key infrastructure for broadband access systems because of huge capacity, small size and lightness, and immunity to electromagnetic interference of optical fibers [1]. FTTH systems providing various kinds of advanced services such as voice, video and data over this single media are connectivity to the customer premises. In order to achieve FTTH realization, a key condition that is further cost reduction of the fiber-optic access network must be satisfied. A key issue for FTTH is the availability of inexpensive optical devices such as beam splitter, MUX/DEMUX, OADM, etc. There is a kind of diffractive optical elements, sometimes called beam splitting or fan-out diffractive optical element [2-5]. It can be dividing the energy

\footnotetext{
${ }^{*}$ Corresponding author. Tel.: +886223626431; fax: +886223631755.

E-mail address: liaoys@ccms.ntu.edu.tw (Y.-S. Liao).
}

of an incoming beam into a number of small beams and applied for sending a signal from one channel, optical fiber or semiconductor laser emitter, into many. The diffractive microlens (DML) is a type of fan-out diffractive optical element. The polymeric DML have advantages of light weight, easy handling, low costs and is very suitable for this demand. Micro-replication technology is a simple and important process for polymer DML. DML patterns are generated by duplicating the surface relief of master mold onto polymer substrate and formed mirror image of templates. It can precisely replicate complex microstructures and is very suitable for mass production of high-quality and low cost DML devices. Regarding to fabrication methods for the DML template, there are essentially two approaches. One is gray-scale lithography to produce continuous surface relief such as laser/electron beam direct writing [6], gray-scale mask photolithography [7]. The other is a binary photolithography to reproduce quasi-continuous surface relief $[2,8]$. The first approach can produce higher diffraction efficiency but it is very expensive or time 
consuming for large arrays. Binary photolithography is very common in semiconductor processes and is usually adopted to fabricate multilevel microstructures. A $2^{M}$-level surface relief using $M$ masks in conjunction with photoresist deposition, exposure, development, as well as etching procedures [9]. However, although the ideal diffraction efficiency of eight-level binary optical lenses reaches $95 \%$, it is difficult to obtain the high-efficiency in practice owing to limitations in the general binary lithography conditions. This in turn is resulted from fabrication errors mainly involving the phase-depth error, the mask-alignment error, and the variation of line width when the mask pattern is transferred to the substrate $[10,11]$. Replication quality also greatly influences optical performance of DML. And it highly depends on polymer behaviors during replication process such as good filling of cavity, good releasing property, high dimension accuracy (no shrinkage). There are two essentially material used in polymeric DMLs replication. One is thermal plastic materials such as PMMA, PC, PET, etc. which are frequently used in injection molding, hot embossing. The other is thermal set materials such as UV curable material which are often used in ultra-violent (UV) imprinting processes. For thermal plastic materials, elevated temperature is required to make thermal plastic polymer become viscous state and it fill cavity under applied pressure. Besides, due to different thermal expansion of template and polymeric DML structure, mechanical locking effect frequently happens after a heat-up and cooldown cycle and it damages DML microstructure in the demolding process $[12,13]$. Comparing to thermal plastic material, liquid type UV curable material has relative good filling property especially in micrometer or less DML structure. With UV irradiation, it also can be cured at room temperature which eliminate microstructure damage problem in demolding step.

In this study, we design and fabricate a polymeric DML to spilt incident light into two individually fibers by the use of UV imprinting process. Replication quality greatly influences optical performance of DML. To eliminate fabrication errors of template, tetraethoxysilane (TEOS) oxide etching is developed and employed in the silicon etching process. A modified imprinting process is proposed in the experiment; a DML structure on the template is dispensed with UV resin and covered with flexible polyethylene terphthalate (PET) film. After curing and demolding, the DML structure will be duplicated on PET substrate. Finally, imprinting quality and optical performance are evaluated and compared with simulation results.

\section{Diffractive microlens pattern}

A DML with two individual focal points can serve as an economical $1 \times 2$ beam splitter coupling the incident light to two individually mounted plastic optical fibers to deliver light signal, schematic illustration are shown in Fig. 1. The computer-generated pattern of the DML with two focal

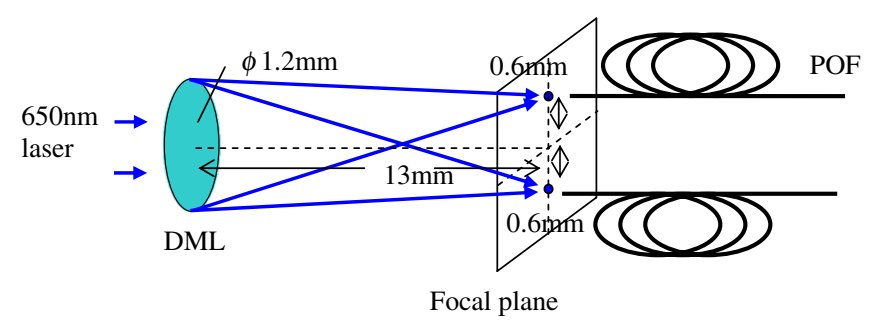

Fig. 1. Schematic of beam splitting DML for coupling $650 \mathrm{~nm}$ laser light into two individually fibers.

points was designed by the optimal-rotation-angle (ORA) method [3,4]. The ORA method is an effective algorithm for designing high-diffraction efficiency patterns based on geometrical analysis in the complex-number plane. It is assumed that the light incident upon the kinoform is monochromatic and its wave fronts are planar. In each discrete point in the diffraction (Fourier) plane, the complex amplitude is the sum of contributions from each pixel in the kinoform plane as given by the discrete Fourier transform.

The input parameters used for the beam splitting lens design were shown in Table 1. An optimum phase solution for the DML with the number of sampling points in the kinoform was numerically found by ORA method. The simulation results of the kinoform and in the diffraction plane are in good agreement with the objective. The simulation results of diffraction efficiency in $1 / e^{2}$ intensity range and spot shape diameter of $1 / e^{2}$ intensity of spot 1 or spot 2 are $57 \%$ and $11.7 \mu \mathrm{m}$, respectively. Generally, the diffraction efficiency of DML amounts to total pixels in the diffraction plane. Here we only summarize the diffraction efficiency of $1 / e^{2}$ intensity. When the planar incident monochromatic lightwave passes through a finite aperture and $100 \%$ efficiency are considered, the ideal diffraction effi-

Table 1

Design specification of DML

\begin{tabular}{ll}
\hline Wavelength & $650 \mathrm{~nm}$ \\
Aperture diameter & $1.2 \mathrm{~mm}$ \\
Number of binary levels & 8 \\
Each etching step & $0.165 \mu \mathrm{m}$ \\
Minimum pixel size & $1 \mu \mathrm{m}$ \\
Number of splits & 2 \\
Energy splitting ratio & $1: 1$ \\
Focal length & $13 \mathrm{~mm}$ \\
Splitting distance between two spots & $1.2 \mathrm{~mm}$ \\
\hline
\end{tabular}

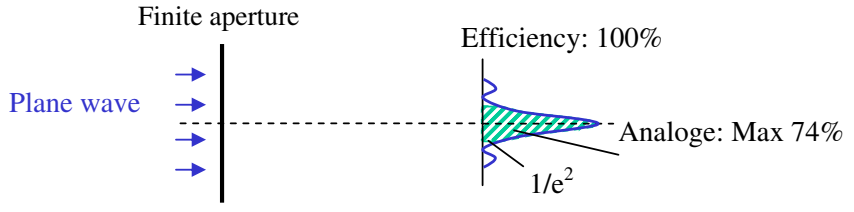

Fig. 2. Schematic of the diffraction efficiency when the planar incident monochromatic lightwave passes through a finite aperture. 
ciency of $1 / e^{2}$ intensity is only $74 \%$. The ideal diffraction efficiency of eight-level binary optical lenses can reach $95 \%$. Therefore, the ideal diffraction efficiency of $1 / e^{2}$ intensity for eight-level binary optical lenses is only $70 \%$. The schematic of diffraction efficiency when the planar incident monochromatic lightwave passes through a finite aperture is shown in Fig. 2.

\section{Fabrication of DML master}

The eight-level DML master was fabricated by repeating a set of photolithography and dry etching processes three times with three different masks. In the experiment, silicon master is the most important fabrication step. The higher optical performance strongly depends on the better characteristics of etched profile such as straight and vertical side wall, low alignment error and high dimension accuracy. To achieve idea profile, a TEOS oxide layer was chosen as etching mask to form a thin protection layer on the side wall during etching, and which ensures vertical side wall slope. To eliminate the misalignment caused by the mechanical tolerance of stage, a self-alignment technique is also realized by using the same TEOS oxide layer. An appropriate mask bias was implanted into the second and third etching masks so as to cover miss alignment. Besides, the size accuracy of pixel is another important factor for DML performance. The most common problem is that it is difficult to have good photolithography pattern on

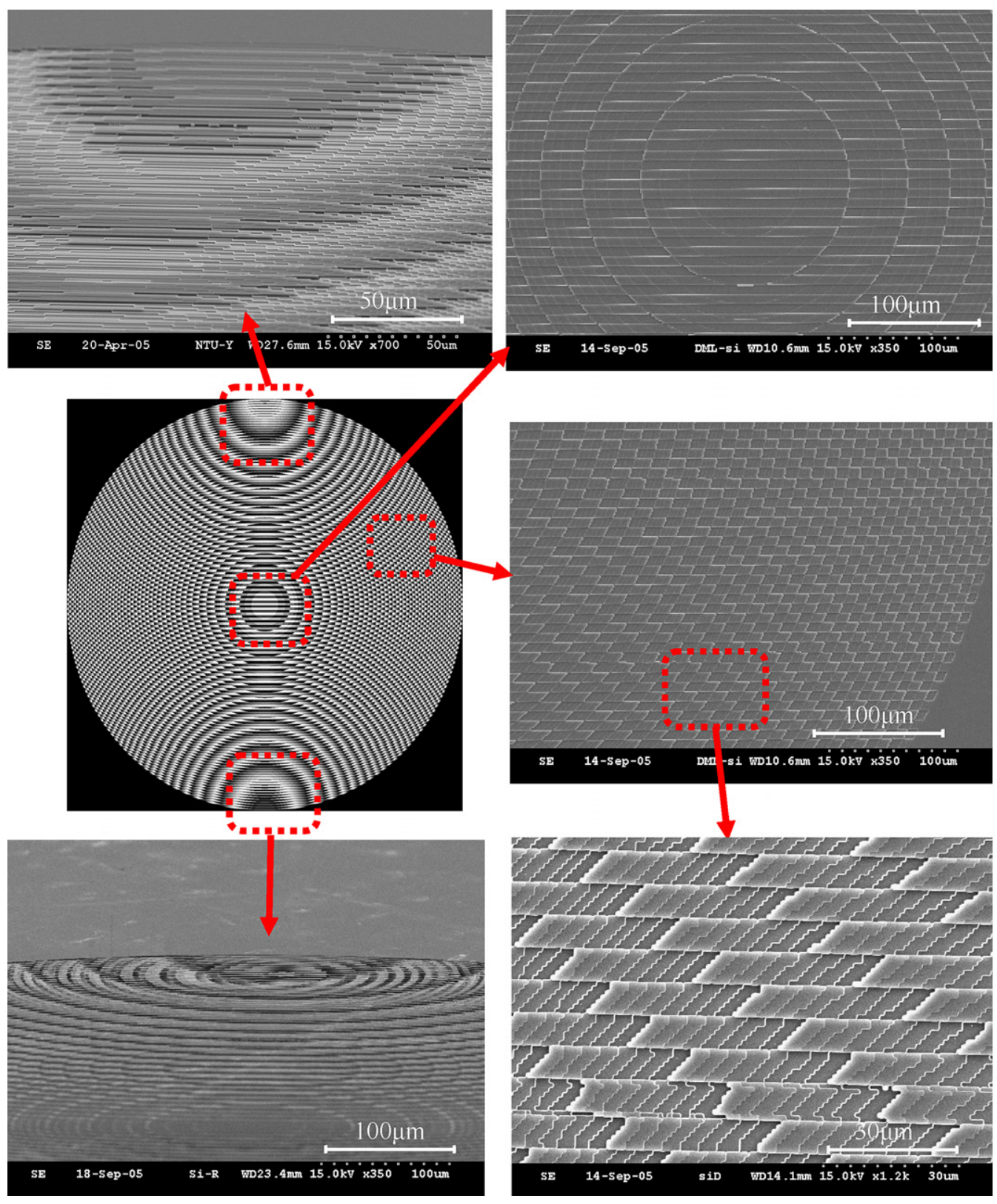

Fig. 3. SEM image of eight-level microstructure on silicon master. 
scraggy profile involving the pixel corner rounding and shape distortion. To eliminate this problem, a designed etching sequence was arranged from the smallest etching depth to the deepest. As a result, the three pieces of mask pattern were successfully transferred onto silicon wafer, and eight-level silicon DML structure was produced as shown in Fig. 3. It is found that a good shape eight levels microstructure is obtained. The alignment error was controlled below $0.1 \mu \mathrm{m}$.

\section{UV imprinting process}

A modified imprinting process by using roller was proposed in the experiment. The imprinting steps can be summarized as shown in Fig. 4: fist the UV curable material was dispensed in liquid form onto the $\mathrm{Ni}$ template to fill concave cavity of DML. For good demolding property, a flexible PET film as DML substrate was bended and placed over dispensed resin to avoid air bubbles trapped in the resin layer. The PET film is transparent for following UV irradiation. Then, the coated template with PET film was laminated by height controlled roller and it moved from one side to the other to remove redundant resin

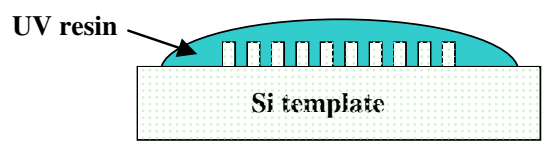

Step 1: Dispense UV

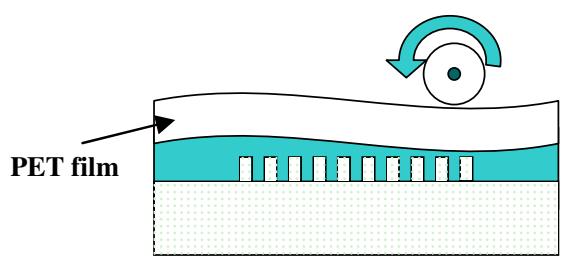

Step 2: Laminated by roller

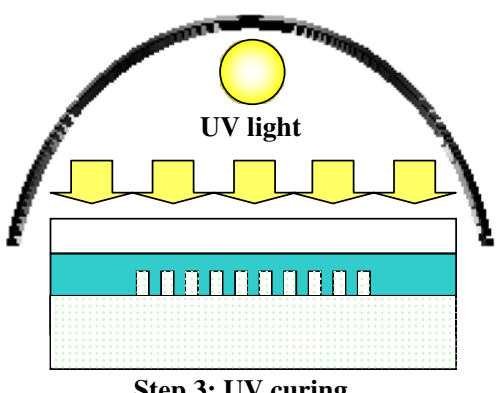

Step 3: UV curing

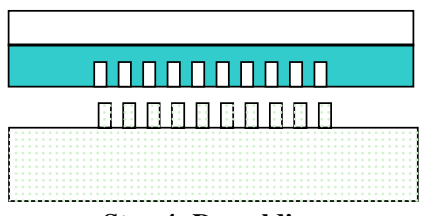

Step 4: Demolding

Fig. 4. Schematic illustration of UV imprinting process. and controlled the UV resin layer thickness. A $200 \mu \mathrm{m}$ thick coated resin layer was obtained. The general wavelength of the UV light of $250-420 \mathrm{~nm}$ was used in the experiment and the exposure intensity was $40 \mathrm{~mW} / \mathrm{cm}^{2}$ which was measured by photometer at $365 \mathrm{~nm}$ wavelength. The sample was exposed to in curing system for $12 \mathrm{~min}$, the resin was solidified at room temperature. After curing step, the flexible PET film with DML structure can be pealed off from DML template and the prototype is shown in Fig. 5a. From SEM image of Fig. 5b, eight-level diffractive structure was duplicated successfully and no significant damage was found. As a result, good replication quality of UV imprinting with flexible substrate was demonstrated.

\section{Results and discussion}

\subsection{Geometrical properties}

A well-defined and highly accurate imprinted etched profile is the foundation for good optical performance. To inspect the fabrication quality, a test pattern was designed to evaluate minimum pixel size, step height, surface roughness and side wall angle. For pixel size analysis, a $1 \mu \mathrm{m}$ continuous step pattern was measured at the edge of each level to inspect the length in both $x$ and $y$ directions. The averaged dimension was $1.02 \mu \mathrm{m}$. And the dimension error was controlled under $10 \%$. It demonstrated good size control in photolithography and UV curing process. For step height analysis, AFM was used to analysis eight levels. The average step height was $0.166 \mu \mathrm{m}$, which showed precise depth control during silicon etching. On the basis of the results, a high-quality imprinted DML with good dimension control was successfully generated.

\subsection{Optical properties}

To inspect the DML device and diffraction efficiency, the measurement setup shown schematically in Fig. 6 was constructed. We obtain the optical intensity distribution using a camera and analyze its optical characteristics using an analyzer. Fig. 7 shows the measurement results of beam splitting image on focal plane and optical intensity profiles of two individual focal points for fabricated polymer DML. The measurement optical properties are listed in Table 2, where the uniformity error of 2 spots $=\mid$ (the spot 2 of peak intensity) - (the spot 1 of peak intensity)|/(the spot 2 of peak intensity) + (the spot 1 of peak intensity)|. In Table 2, it is noted that the errors of all optical properties are small. The focal lengths for both spots are about $12.8 \mathrm{~mm}$. The distance between spots 1 and 2 is $1.18 \mathrm{~mm}$ (designed value is $1.2 \mathrm{~mm}$ ). The spot diameters of $1 / e^{2}$ intensity are smaller than $13 \mu \mathrm{m}$. The diffraction efficiency is $51 \%$ (designed value is $57 \%$ ) and the uniformity error is only $1.5 \%$. 
a

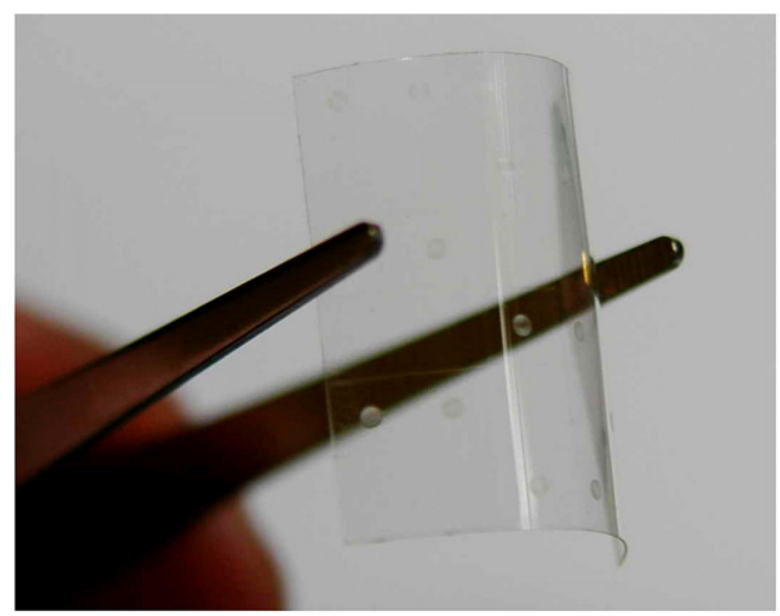

b

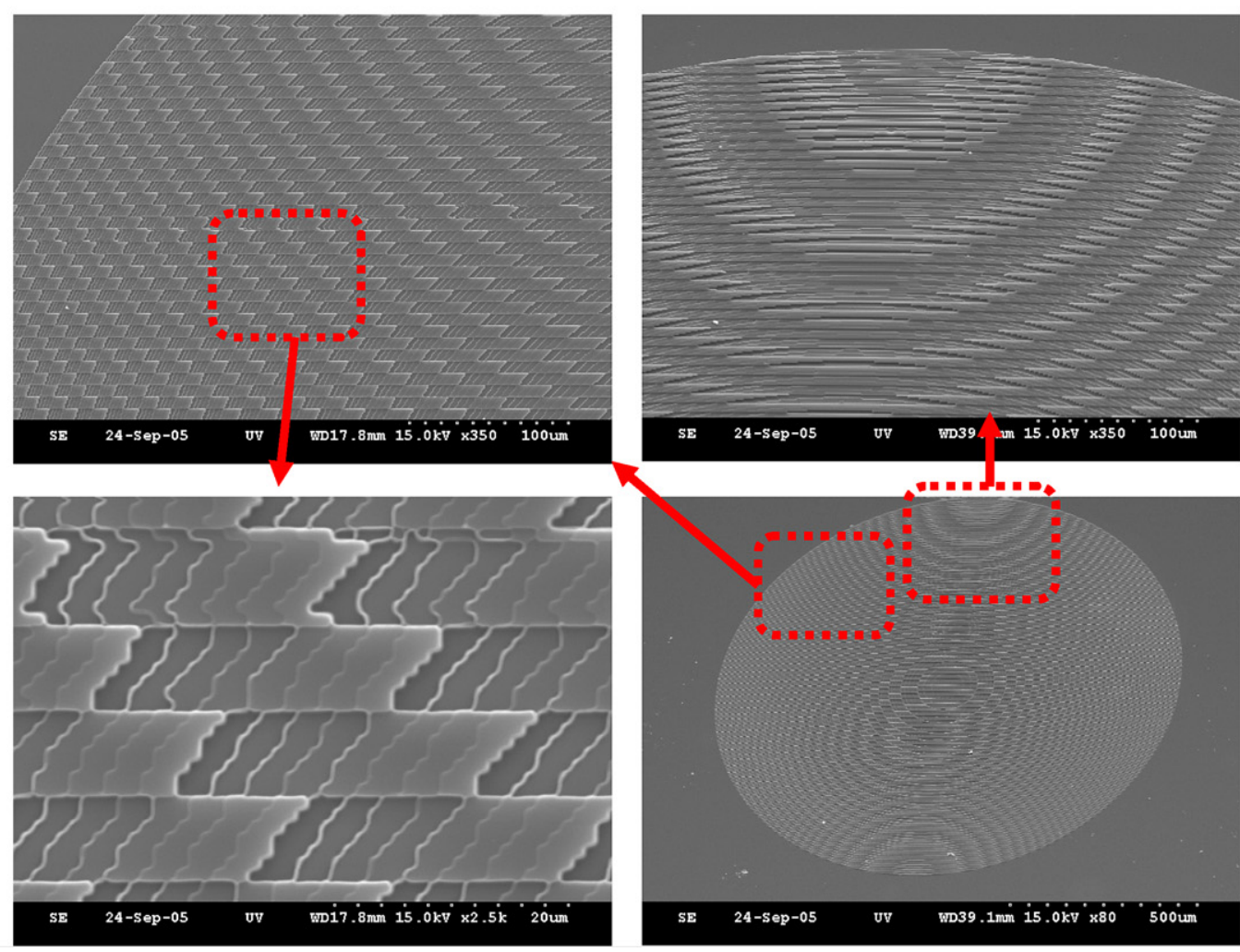

Fig. 5. (a) Photograph of imprinted DML on transparent and flexible PET substrate. (b) SEM image of UV imprinted structures and local area.

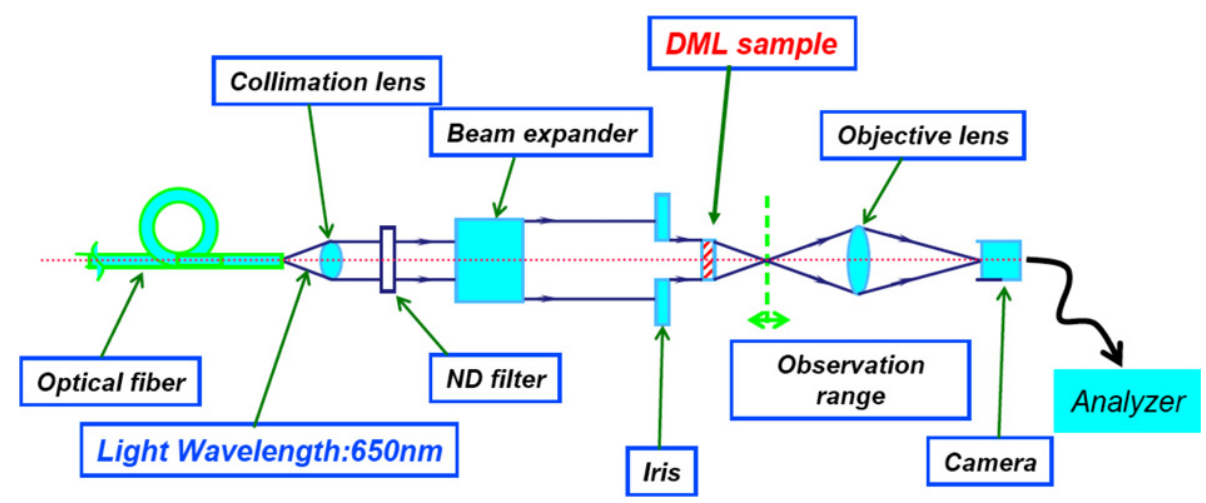

Fig. 6. Schematic illustration of optical measurement setup. 
a
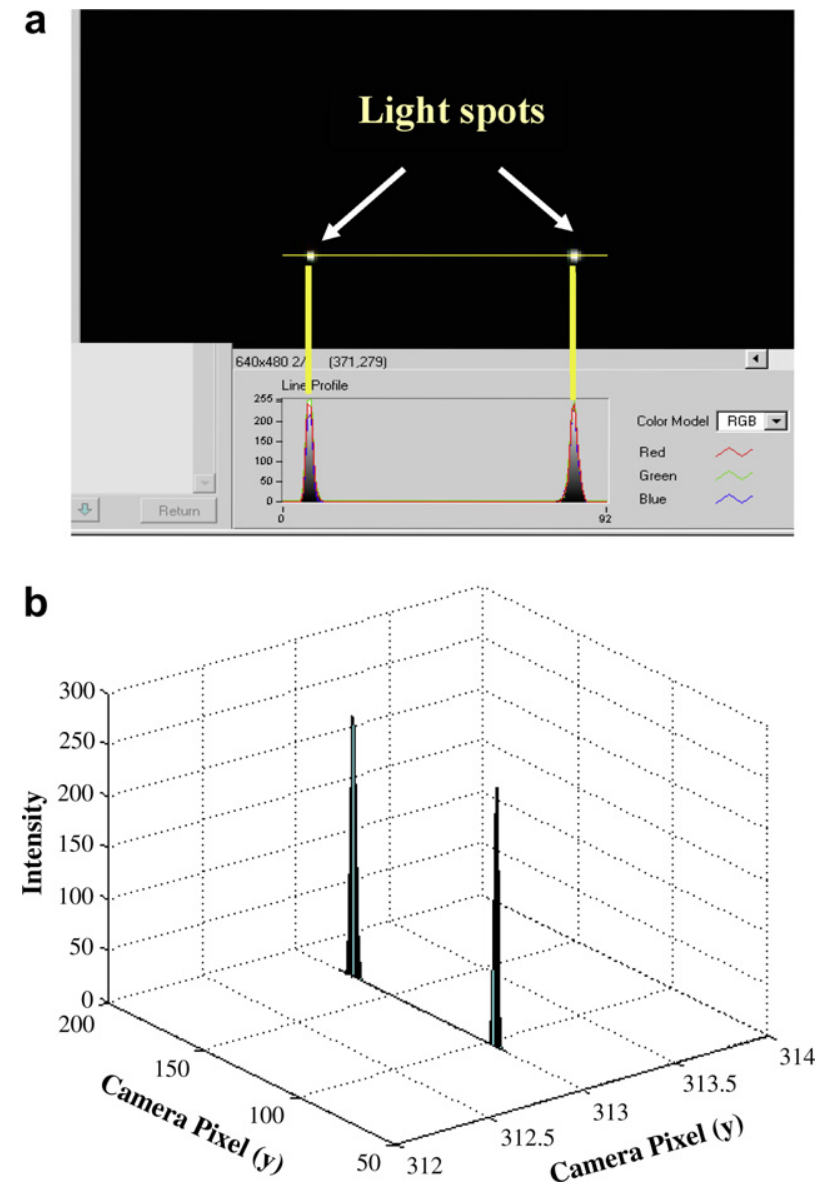

Fig. 7. Measurement result for DML. (a) Camera image on focal plane; (b) optical intensity profiles of two individual focal points.

Table 2

Measurement results and errors of optical properties

\begin{tabular}{lll}
\hline Item & \multicolumn{2}{l}{ Measurement (design) } \\
\cline { 2 - 3 } & Spot 1 & Spot 2 \\
\hline Distance between 2 spots & $1.18 \mathrm{~mm}(1.2 \mathrm{~mm})$ & \\
Diffraction efficiency & $51 \%(57 \%)$ & \\
Focal length & $12.7 \mathrm{~mm}(13 \mathrm{~mm})$ & $12.7 \mathrm{~mm}(13 \mathrm{~mm})$ \\
Spot diameters of $1 / e^{2}$ intensity & $<13 \mu \mathrm{m}(11.7 \mu \mathrm{m})$ & $<13 \mu \mathrm{m}(11.7 \mu \mathrm{m})$ \\
Uniformity error of two spots & $1.5 \%$ & \\
\hline
\end{tabular}

\section{Conclusions}

A polymeric DML for coupling the incident light to two individually plastic optical fibers was designed and fabricated by imprinting process. The DML pattern of this dual-focal-point DML was designed with an optimal rotation-angle and realized by template fabrication and UV imprinting. TEOS oxide etching was developed and employed in the template fabrication process to minimize the related processing errors such as misalignment and dimension accuracy. A modified imprinting process is proposed in the experiment; DML with flexible PET substrate was imprinted by roller. After curing and demolding, the DML structure was successfully duplicated on PET substrate. The prototype was inspected and to confirm the optical properties and the results showed that the diffraction efficiency is $51 \%$ (designed value is $57 \%$ ) and the uniformity error is only $1.5 \%$.

\section{Acknowledgements}

The authors would like to thank Dr. T.C. $\mathrm{Wu}, \mathrm{Mr}$. M.Y. Liu and Mr. C.K. Mu at MIRL of ITRI, Taiwan for their guidance and assistance.

\section{References}

[1] I. Yamashita, T. Matsumoto, N. Shibata, Opt. Fiber Technol. 4 (1998) 189.

[2] M.P. Dames, R.J. Dowling, P. McKee, D. Wood, Appl. Opt. 30 (1991) 2685.

[3] J. Bengtsson, Appl. Opt. 33 (1994) 6879.

[4] J. Bengtsson, Appl. Opt. 36 (1997) 8435.

[5] M.R. Taghizadeh, P. Blair, B. Layet, I.M. Barton, A.J. Waddie, N. Ross, Microelectron. Eng. 34 (1997) 219.

[6] M.T. Gale, M. Rossi, J. Pedersen, H. Schutz, Opt. Eng. 33 (1994) 3556.

[7] Y. Oppliger, P. Sixt, J.M. Stauffer, J.M. Mayor, P. Regnault, G. Voirin, Microelectron. Eng. 23 (1994) 449.

[8] G.J. Swanson, W.B. Weldkamp, High-efficiency, multi-level, diffractive optical elements, US Patent 4,895,790, January 23, 1987.

[9] J.R. Leger, M.G. Moharam, T.K. Gaylord (Eds.)Appl. Opt. 34 (1995) 2399.

[10] U. Krackhardt, N. Streibl, J. Schwider, Optic 95 (1994) 37.

[11] Y. Unno, Appl. Opt. 37 (1998) 3401.

[12] N. Roos, H. Schulz, L. Bendfeldt, M. Fink, K. Pfeiffer, H.-C. Scheer, Microelectron. Eng. 61-62 (2002) 399.

[13] H. Becker, U. Heim, Sensor Actuator A: Phys. 83 (2000) 130 